\title{
Enhanced recovery clinical education programme improves quality of post- operative care
}

Ruth McDonald

University College Hospital London

\begin{abstract}
Quality is the driving principle of Enhanced Recovery (ER). It improves the patient experience by getting patients better sooner and changes clinical practice to make care safer and more efficient. As a consequence of ER patients spend less time in hospital.

A successful ER programme began to fail after organisational restructuring and staff changes. Patients did not meet their ER goals and length of stay (LOS) increased. An ER nurse was appointed to get the programme back on track.

This involved a multidisciplinary approach to an ER clinical education programme. The programme aimed to develop knowledge of the physiology of post-operative recovery and the evidence underpinning the interventions required. This was considered crucial to secure longer term staff engagement while avoiding unthinking protocol driven compliance. Success of the education programme was measured by improved outcomes in patient LOS and readmission statistics.

During the four months of the clinical education programme there were no significant changes in monthly LOS. At six months post implementation of the programme there was a reduction in LOS of 0.6 days compared to the previous six months. At 12 months there was a reduction in 1.1 days compared with previous 12 months. There was a mean reduction of 28 day readmissions for all elective gynaecology surgery of 1.1 patients per month in the 12 months post programme implementation compared to the 12 months before. Delivering a multidisciplinary participatory education programme improved overall understanding of ER, and achieved sustained improvement in ER for patient benefit.
\end{abstract}

\section{Problem}

In 2011 a successful enhanced recovery (ER) programme for gynaecology was launched as a six month project in a NHS teaching hospital. Organisational restructure and staff changes in 2012 led to failure of the programme: ER post-operative goals were not achieved and length of stay increased. In 2013 an ER nurse was appointed to lead the programme.

\section{Background}

Enhanced recovery (ER) programmes aim to deliver an optimal surgical pathway designed to minimise the overall physiological and psychological impact of the surgical procedure helping patients recover sooner.[2,5,6]

An ER pathway can be divided into three main elements: preoperative patient preparation; peri-operative interventions; and postoperative rehabilitation. Prior to the clinical education programme launch, pre-operative and peri-operative elements had been developed and introduced.[4] A generic patient information booklet covering the whole elective pathway was posted to all elective gynaecology surgery patients with their dates for pre-operative assessment clinic and surgery. In addition, the ER nurse trained all pre-operative assessment nurses to educate patients to assist in their own recovery and preparations for admission and discharge.
ER peri-operative practice successfully introduced in 2011 involves the patient meeting with their consultant surgeon on the morning of their surgery to confirm their consent for their procedure. Laparoscopic surgical interventions are used whenever possible, and no nasogastric tubes or abdominal drains are used routinely. Temperature is controlled and antiemetics, analgesia, and prophylactic antibiotics are administered. Goal directed fluid therapy is used in the major open cases and a zero balance is aimed for in laparoscopic cases.[1,4] Carbohydrate loading is not formally introduced, though patients are advised on minimum starvation times and that clear fluid energy drinks, Powerade or Gatorade, are beneficial to drink two hours before surgery.

The primary post-operative elements of ER are:
1. Early mobilisation
2. Early nutrition
3. Pro-active management of pain and nausea
4. Restricted intravenous fluid administration. $[1,4,7]$

A team approach to ER is essential to its sustainability and success. It cannot be implemented by an enthusiastic clinician alone but is reliant on clinical engagement from surgical, nursing, allied health professionals and anesthetic teams.[1] Even the simple changes in practice required to deliver the post-operative rehabilitation elements, is acknowledged [3] to represent a fundamental change in practice and can therefore be difficult to 
achieve. Central to the success of an ER programme is the education of staff, patients, and relatives.[6] The focus of this project is the multidisciplinary education of ward staff delivering an ER programme, since baseline clinical observation had identified the programme was failing.

\section{Baseline measurement}

Clinical observations were carried out to ascertain adherence to enhanced recovery (ER) post-operative goals and measurement of inpatients length of stay (LOS). LOS reduction is not the primary goal of ER but it is a useful marker for the effectiveness and consistency of a programme.[6] LOS data is collected centrally for all patients within the hospital. Utilising this data enabled accurate comparison with historical data and trends as the ER programme evolved.

Since the organisational restructure in 2012, gynaecology patients were split over two wards: a short stay ward and a mixed speciality acute surgical ward. To compare LOS for similar procedures on both wards, all procedures involving hysterectomy (general gynaecology and gynaecology oncology) were selected.

Readmission data within 28 days for all gynaecology procedures was taken from CHKS live (a healthcare intelligence provider). This was monitored to ensure a reduction in LOS did not increase readmission rates. As a baseline, LOS and 28 day readmission data was taken from previous ER implementation year 2011/12 and from the year of organisational restructure 2012/13 (see figure 1).

Clinical observations identified:

1. Patients were not mobilising early, this was often due to uncontrolled pain

2. Post-operative pain management was suboptimal

3. Staff were unsure what patients should eat after surgery

4. Intravenous fluids were continuing until a bag had finished rather than when medically indicated.

See supplementary file: ds5033.pptx - "Baseline LOS \& readmissions"

\section{Design}

The aim was to develop and deliver a clinical education programme to reach as many ward based nurses and healthcare assistants as possible with minimal impact on clinical duties and staffing resources. Scheduling study days was not considered cost effective and the ward would only be able to release a small number of staff each month.

The location, timing and frequency of teaching sessions were arranged with the ward sister and practice educator to minimise disruption to clinical care. $1 \mathrm{pm}-2 \mathrm{pm}$ was chosen as the teaching window as all lunchtime medications had been administered, patients rest period had started and staff lunches start at $2 \mathrm{pm}$, so the ward was fully staffed.
A three month teaching programme was developed with input from the full range of involved clinicians including consultant surgeons, physiotherapists, the acute pain team, and dieticians, to address the elements of Enhanced Recovery (ER) which were observed to be suboptimal. All information for the teaching sessions would be displayed on the ER notice board in the central corridor of the ward and each 15 minute teaching session would be delivered around this board. The content of the teaching programme aimed to develop knowledge of the physiology of post-operative recovery and the evidence underpinning the interventions required.

Physiotherapists and acute pain nurses engaged ward staff in their patient assessments and interventions to develop confidence in mobilisation and pain assessments during the three education sessions on post-operative rehabilitation. In addition, dieticians jointly delivered teaching with the ER nurse and ran interactive supplement tasting sessions for nursing and medical staff. As the teaching was being delivered on a mixed urology and gynaecology ward the urology advanced nurse practitioner was consulted and agreed to the teaching plan and session content to be applied to urology ER patients.

\section{Strategy}

Improvement (Plan Do Study Act - PDSA) cycle 1: A three month clinical education programme was planned and delivered to ward nurses and healthcare assistants on a mixed speciality acute surgical ward. Three topics were covered (one each month) and the relevant topics' teaching materials were displayed on the ward ER notice board each month. Action at the end of cycle 1 was to continue monitoring LOS on the acute surgical ward and to run the programme on the short stay surgical ward.

Improvement (PDSA) cycle 2: This was unsuccessful from an early stage (step 2, "Do"). The ward was short staffed and due to the unpredictable work flow on the short stay ward, teaching sessions were regularly cancelled (only two teaching sessions ran and the rest were cancelled). The education programme stopped for review after one month. The action at the end of this cycle for the short stay ward was to design a study day away from the ward when staffing levels improved.

\section{Results}

Improvement (PDSA) cycle 1: the monthly data on LOS, showed minor fluctuations above and below the baseline (Jan 2014) in the first three months. It became apparent that trend analysis over a longer period would be a more appropriate method to evaluate the impact of the clinical education inputs.

At six months post implementation of the programme there was a reduction in LOS of 0.6 days compared to the 6 months before. At 12 months there was a reduction in 1.1 days compared with the 12 months before, for all procedures involving hysterectomy (figure 5). There was a mean reduction of 28 day readmissions for all elective gynaecology surgery of 1.1 patients per month in the 12 months post programme implementation compared to the 12 months before 
(figure 6).

A total of 14 sessions were held and a total of 62 staff attended. The programme ran over 4 months instead of the planned 3 months due to session cancelled by the ward and annual leave.

Clinical observations after the ER education programme:

1. The majority of patients were sitting out of bed for breakfast

2. Physiotherapist reported a reduction in patients who were unable to participate in physiotherapy due to pain

3. Ward staff reported increased confidence in advising patients on post-operative diet

4. Intravenous fluids were being stopped earlier.

Improvement (PDSA) cycle 2 was unsuccessful from an early stage and therefore data was not analysed as the project had not passed the initial stage.

See supplementary file: ds5034.pptx - "Readmissions and LOS April 13 - Jan 15"

\section{Lessons and limitations}

Teaching around a notice board in the middle of a ward environment was challenging. Staff could easily be distracted or occasionally have to answer call bells. Keeping the groups small (four staff members) and the sessions to 15 minutes was important and helped mitigate against these distractions. Although challenging, this peripatetic teaching approach was efficient and effective and will be used again. Staff did engage in the sessions and agreed a set of achievable actions at the end of the sessions. Delivering short 15 minute sessions in a busy clinical environment did however limit the amount of detail provided.

The technique worked best on the acute surgical ward as the work flow is more predictable than on the short stay ward.

Attempts to run the education programme on the short stay ward (PDSA cycle 2) in the same format has been less successful. The short stay ward did not have the natural patient rest period for patients as the patient flow to and from the ward is fast. Therefore the teaching sessions were regularly cancelled due to workload. A period of one-to-one teaching was attempted but this was very time consuming and was not an efficient use of the Enhanced Recovery (ER) nurse resource. A series of women's health study days have now been designed by the gynaecology advanced nurse practitioner and this has an ER section. The short stay ward is able to release a greater number of staff to these protected non-clinical sessions. These sessions are also resource efficient as they cover a wide range of topics and updates in women's health nursing.

Involving the multidisciplinary team in planning and delivery aimed to improve the overall understanding of ER and help achieve sustainability of the programme. Collaboration with Urology supported the programme's principles of best surgical practice for all surgical patients.
Since the completion of the education programme there has been a high turnover of staff on the acute surgical ward and this once again threatens the sustainability and effectiveness of ER implementation.[5] The clinical education programme will be repeated annually to counter these negative effects. This will address the high turnover of staff and provides a refreshing opportunity for the clinical multidisciplinary team to come together for continual professional development. This enhances joint ownership and responsibility for the success of the ER programme and all new staff across the different disciplines will be introduced to the local ER pathway at induction.

By continuing to analyse the data over a longer period of time it was possible to monitor if the change was being sustained.

\section{Conclusion}

The Enhanced Recovery (ER) clinical education programme improved quality of post-operative care, leading to reduced length of stay with no increase in 28 day readmission rates.

Providing ward staff with the evidence underpinning the ER pathway and involving the multidisciplinary team helped achieve sustainability of the ER programme. Having a dedicated ER nurse to coordinate this team approach and all other aspects of the programme is integral to successful implementation of an ER package.[7]

\section{References}

1. Department of Health. Quality and Productivity. Enhanced recovery for elective surgery. 2009 https://arms.evidence.nhs.uk/resources/qipp/29459/attachm ent

2. Grace C. Fitter, faster: improved pathways speed up recovery. Health Service J 2011 May 5; 28-30.

3. Melnyk M, Casey RG, Black P, Koupparis AJ. Enhanced recovery after surgery (ERAS) protocols: Time to change practice? Can Urol Assoc J 2011;5(5):342-8.

4. Enhanced Recovery Partnership. Enhanced recovery care pathway: A better journey for patients seven days a week and better deal for the NHS. Progress review (2012/13) and level of ambition (2014/15). NHS Improving Quality. 2013 Nov.

5. Paton F, Chambers D, Wilson P, et al. Effectiveness and implementation of enhanced recovery after surgery programmes: a rapid evidence synthesis. BMJ Open 2014;4:e005015. doi:10.1136/bmjopen-2014-005015

6. Dutton TJ, Daugherty MO, Mason RG and McGrath JS. Implementation of the Exeter Enhanced Recovery Programme for patients undergoing radical cystectomy. BJU Int 2014; 113: 719-25

7. Khan s, Gatt M, Horgan A, Anderson I and MacFie J. Guideline for implementation of enhanced recovery protocols (2009) Issues in professional practice. Association of Surgeons of Great Britain and Ireland. 


\section{Declaration of interests}

None declared.

\section{Acknowledgements}

With thanks to Miss Nicola MacDonald (Consultant Gynaecological Oncologist), Esther Kuria (Gynaecology Advanced Nurse Practitioner), Randall Stricker and Rozanna King (UCLH Acute Pain Team), Helen Mellersh and Grace Sheppard (UCLH Women's Health Physiotherapy),

Ellie Allen, Kassie Montanheiro, Jo Eady and Tiziana Midolo (UCLH dietitians),

Gillian Basnett (Urology Advanced Nurse Practitioner), and Dr. Anne Lanceley (Senior Lecturer, the UCL EGA Institute for Women's Health).

\section{Ethical approval}

Ethical approval was not sought for this study as it was a re-launch of a programme that had been in practice for all elective

Gynaecology patients for 2 years. 